\title{
Adhesion signaling promotes protease-driven polyploidization of glioblastoma cells
}

\author{
JAVIER MERCAPIDE and AURELIO LORICO \\ Cancer Research Center, Roseman University of Health Sciences, Las Vegas, NV, USA
}

Received June 30, 2014; Accepted August 8, 2014

DOI: $10.3892 / \mathrm{ijmm} .2014 .1913$

\begin{abstract}
An increase in ploidy (polyploidization) causes genomic instability in cancer. However, the determinants for the increased DNA content of cancer cells have not yet been fully elucidated. In the present study, we investigated whether adhesion induces polyploidization in human U87MG glioblastoma cells. For this purpose, we employed expression vectors that reported transcriptional activation by signaling networks implicated in cancer. Signaling activation induced by intercellular integrin binding elicited both extracellular signal-regulated kinase (ERK) and Notch target transcription. Upon the prolonged activation of both ERK and Notch target transcription induced by integrin binding to adhesion protein, cell cultures accumulated polyploid cells, as determined by cell DNA content distribution analysis and the quantification of polynucleated cells. This linked the transcriptional activation induced by integrin adhesion to the increased frequency of polyploidization. Accordingly, the inhibition of signaling decreased the extent of polyploidization mediated by protease-driven intracellular invasion. Therefore, the findings of this study indicate that integrin adhesion induces polyploidization through the stimulation of glioblastoma cell invasiveness.
\end{abstract}

\section{Introduction}

Polyploidization contributes to genetic instability in cancer by causing the unequal distribution of chromosomes in cell divisions (1-3). That linkage supports a role of mechanisms that augment the number of tumor genomes per cell, e.g., cell fusion and failed mitosis/absence of cell division following genome replication in increasing the degree of aneuploidy (2).

Correspondence to: Dr Aurelio Lorico, Cancer Research Center, Roseman University of Health Sciences, 10530 Discovery Drive, Las Vegas, NV 89135, USA

E-mail: alorico@roseman.edu

Abbreviations: ERK, extracellular signal-regulated kinase; MMP, matrix metalloproteinase; $\mathrm{Nr}-\mathrm{CAM}$, neuronal cell adhesion molecule; PBS, phosphate-buffered saline

Key words: cell fusion, genome duplication, glioblastoma, integrin signaling, invasion, polyploidization
Intriguingly, upon increasing the content of DNA by heterotypic cell fusion, cancer cells undergo large-scale changes in gene expression that induce progression $(4,5)$. These findings implicate cell fusion as a mechanism of cancer cell progression towards highly malignant phenotypes, thus suggesting that certain fusogenic mechanistic underpinnings of cells may be a feature of carcinogenic transformation. Molecular studies have elucidated sequential cell-cell fusion steps in fusogenic cells that contribute to mammalian development, tissue repair and homeostasis (6-8), and have provided experimental support for the possibility of an association between cell fusion and cancer cell transformation (6). However, the molecular determinants of cell-cell fusion in cancer remain unknown.

In a previous study, in a set of human cancer cell lines, we found a correlation between the proportion of cells with genome duplication and the proportion of cells invading through proteolytic degradation (9). Thus, the contribution of invasion to polyploidization indicated that tumor-genome duplication was mediated by intracellular invasion, which led us to hypothesize that adhesion signaling may induce polyploidization in cancer cells. In this study, employing expression vectors of a reporter array for transcription factors of signaling networks implicated in cancer, we pinpointed pathways of signal transduction in glioblastoma cells to investigate whether adhesion signaling determines the frequency of polyploidization.

\section{Materials and methods}

Reagents. Integrin- $\beta 1$ polyclonal antibody was obtained from Cell Signaling Technology, Inc. (Danvers, MA, USA); antibody for hyaluronan receptor (clone G44-26) was from BD Biosciences (San Jose, CA, USA); antibody for neuronal cell adhesion molecule (Nr-CAM) (clone A27), marimastat and matrix metalloproteinase (MMP) inhibitor II were purchased from Santa Cruz Biotechnology, Inc. (Santa Cruz, CA, USA); non-specific antibodies were obtained from Rockland Immunochemicals, Inc. (phycoerythrin-conjugated) (Gilbertsville, PA, USA), and Santa Cruz Biotechnology, Inc.

Tissue culture. The cell cultures were maintained at $37^{\circ} \mathrm{C}$ in a humidified atmosphere containing $5 \% \mathrm{CO}_{2}$. U87MG cells (American Type Culture Collection, Manassas, VA, USA) were maintained in minimum essential medium (Mediatech, Herndon, VA, USA) supplemented with $10 \%$ fetal bovine serum 
(Atlanta Biologicals, Inc., Flowery Branch, GA, USA), $2 \mathrm{mM}$ L-glutamine (HyClone, Logan, UT, USA), 50 U/ml penicillin and $50 \mu \mathrm{g} / \mathrm{ml}$ streptomycin (both from Lonza, Rockland, ME, USA). Neural progenitor cells from newborn C57BL/6 mice (Harlan Laboratories, Indianapolis, IN, USA) were obtained, after euthanasia following a protocol approved by the Animal Care and Use Committee of our Institution, through incubation of sections of the forebrain in neurobasal medium supplemented with B27 (both from Invitrogen Life Technologies, Carlsbad, CA, USA), $20 \mathrm{ng} / \mathrm{ml}$ epidermal growth factor, $10 \mathrm{ng} / \mathrm{ml}$ basic fibroblast growth factor (both from R\&D Systems, Minneapolis, MN, USA), $2 \mathrm{mM} \mathrm{L}$-glutamine, $50 \mathrm{U} / \mathrm{ml}$ penicillin and $50 \mu \mathrm{g} /$ $\mathrm{ml}$ streptomycin. Fluorescent marker-expressing cells, obtained by clonal expansion (U87MG cells) or fluorescence-activated cell sorting (neural progenitor cells) after transduction with retroviral vectors as previously described (10), were co-cultured in neurobasal medium. Tissue culture trypsinization was carried out with Hank's balanced salt solution containing $0.05 \%$ trypsin (Mediatech), and terminated by the addition of serum-supplemented culture medium (U87MG cells) or by dilution (neural progenitor cells).

Transduction with luciferase reporter vector. Stably transduced reporter cells were obtained by cell culture incubation in medium containing lentiviral particles with luciferase expression vector (Cignal Finder Cancer Reporter Array; SABiosciences, Frederick, MD, USA), and the selection of the population of transduced cells through the addition of puromycin $(0.5 \mu \mathrm{g} / \mathrm{ml}$; Sigma-Aldrich, St. Louis, MO, USA).

Luciferase assay. Luciferase expression by cells transduced with reporter vector, containing either non-inducible TATA-box promoter or TATA-box promoter joined to one of 10 different transcriptional-response elements, was quantified in 96-well plate adherent cultures. For this purpose, a 1:1 mixture of ONE-Glo ${ }^{\mathrm{TM}}$ Luciferase reagent (Promega Corp., Madison, WI, USA) and cell culture medium supplemented with $2 \%$ fetal bovine serum was added to the cells ( $50 \mu \mathrm{l} /$ cell culture) and, $10 \mathrm{~min}$ later, the luminescence of the resulting cell lysate was measured in a Turner Biosystems 20/20 luminometer (Turner Biosystems, Inc., Sunnyvale, CA, USA).

Determination of cell density-dependent transcriptional activation. In a series consisting of 5 individual cultures of lentiviral vector-transduced cells set up with cell densities decreasing, from 59,200 cells $/ \mathrm{cm}^{2}$, by a 2 -fold serial dilution factor, luciferase reporter gene expression was determined after $48 \mathrm{~h}$ from cell seeding, at which time cell confluency was expected to be 64, 32, 16, 8 and 4\% (control) (based on $34 \mathrm{~h}$ doubling time and 250,000 cells $/ \mathrm{cm}^{2}$ in a confluent culture). The fold change in cell expression of reporter was determined by dividing the value of luminescence by the average value of the corresponding control cultures (4\% cell confluency) and by the corresponding dilution. A statistical threshold for transcriptional activation was established from all the average ratios obtained as the median $\pm \mathrm{SD}$. Average ratios above the threshold were considered indicative of promoter activation.

Protein determination. Total protein amount per cell culture was estimated using the BCA protein assay kit (Pierce
Biotechnology, Inc., Rockford, IL, USA), after rinsing each culture with phosphate-buffered saline (PBS) and lysing the cells with cold lysis buffer ( $\mathrm{pH}$ 7.4) containing $20 \mathrm{mM}$ Tris, $0.5 \%$ Triton X-100, $150 \mathrm{mM} \mathrm{NaCl}$ and protease inhibitor cocktail (1:100; EMD Millipore, Billerica, MA, USA).

Plate coating. Untreated polystyrene plates were coated with the adhesion protein, retronectin (Takara, Otsu, Japan). Retronectin was added at different dilutions in PBS to non-tissue culture-treated plates (BD Biosciences), removing the solutions after $2 \mathrm{~h}$ of coating at room temperature. The coated surfaces were rinsed with PBS before seeding the cells. The retronectin coating concentrations were determined as indicated by the manufacturer.

Cell DNA content distribution analysis. Cell DNA content was quantified by measuring the emission intensity of fluorescent dye-stained DNA in a flow cytometer (Guava EasyCyte ${ }^{\mathrm{TM}}$ Plus System; EMD Millipore). For this purpose, the cell culture was trypsinized and the cell suspension passed through a cell strainer $(70 \mu \mathrm{m})$ (BD Biosciences). After blocking trypsinization, $2 \times 10^{4}$ cells were centrifuged and resuspended in $0.5 \mathrm{ml}$ of PBS. Using a fine tip pipette, the suspension was drawn up and expelled repeatedly. Subsequently, upon the addition of a 10-fold larger volume of buffer, the cells were centrifuged and resuspended in $200 \mu \mathrm{l}$ of PBS. The suspension was added drop by drop to $10 \mathrm{ml}$ of ice-cold $70 \%$ ethanol for cell fixation. The fixed cells were centrifuged at high speed $(450 \mathrm{xg})$, resuspended in PBS containing 1\% bovine serum albumin, re-centrifuged and resuspended in $200 \mu 1$ of Guava cell cycle reagent (EMD Millipore). The distribution of propidium iodide-stained cells was determined using Guava CytoSoft 5.3 software (Guava Technologies, Inc., Hayward, CA, USA).

Estimation of the fraction of binucleated cells. The fraction of binucleated cells over the total cells was quantified in 3-day cultures on a retronectin-coated surface (seeding density, 15,000 cells $/ \mathrm{cm}^{2}$ ). Each culture was trypsinized and re-seeded in a regular culture well and, soon after attachment, the cells were fixed with $4 \%$ p-formaldehyde and stained with 4',6-diamidino-2-phenylindole (1:1,000; Sigma-Aldrich). Binucleated cells were counted in randomly selected areas using an Olympus CKX41 fluorescence microscope (Olympus, Center Valley, PA, USA).

Quantitative assessment of receptor expression. The cell surface expression of hyaluronan receptor was quantified by flow cytometric analysis. To this aim, $2 \times 10^{4}$ cells/sample were resuspended in PBS containing $0.1 \%$ bovine serum albumin and, then, half of the cells were incubated for $30 \mathrm{~min}$ at $4^{\circ} \mathrm{C}$ with phycoerythrin-conjugated antibody for hyaluronan receptor and the other half with phycoerythrin-conjugated non-specific antibody (antibody concentration, $0.5 \mu \mathrm{g} / \mathrm{ml}$ ). Following incubation with antibody, the cells were washed twice and resuspended in $200 \mu$ of PBS for the analysis of fluorescence intensity in a Guava flow cytometer.

Statistical analysis. The Student's t-test was used to determine p-values. $p<0.05$ was considered to indicate a statistically significant result. 


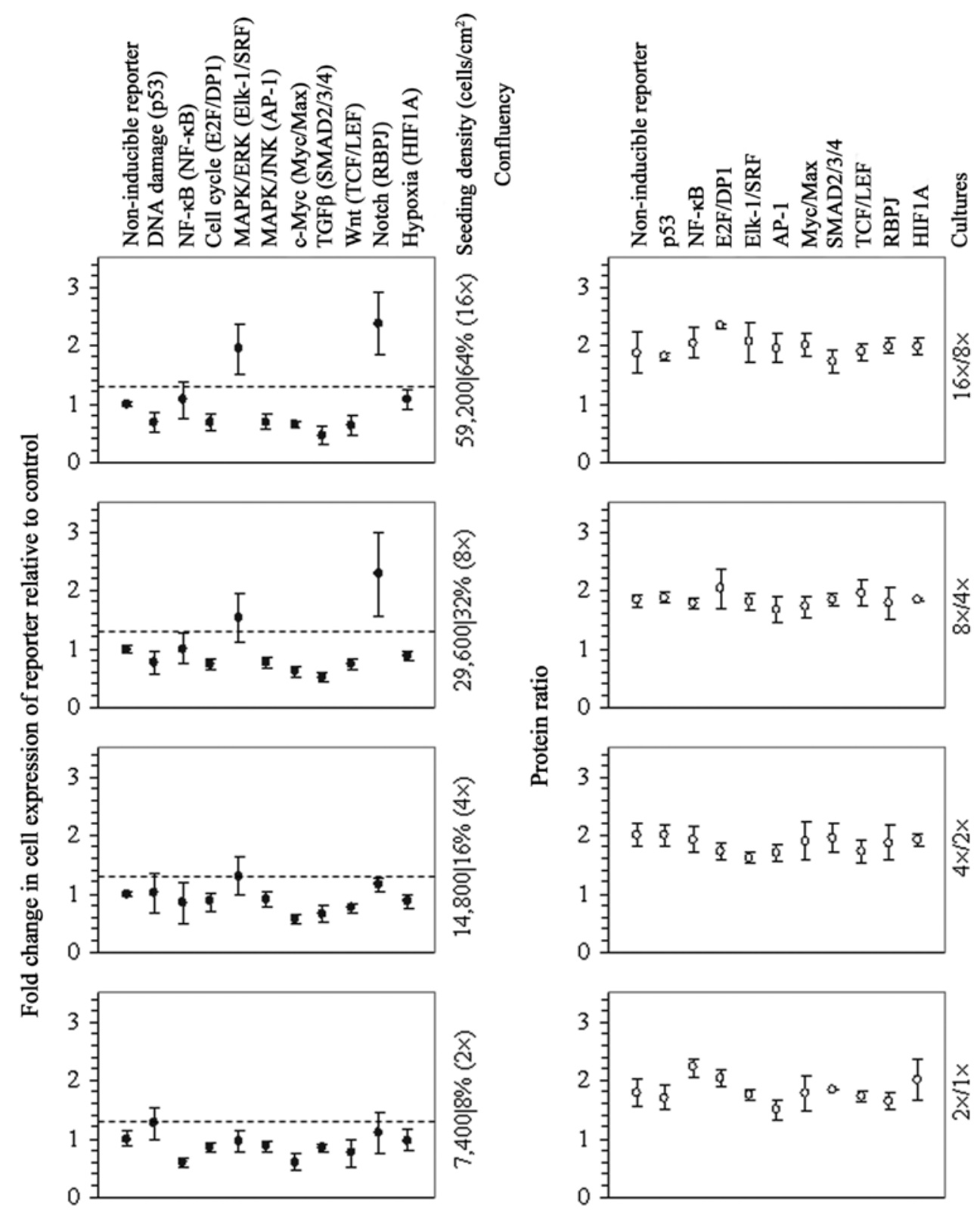

Figure 1. Intercellular adhesion activates both extracellular signal-regulated kinase (ERK) and Notch target transcription in U87MG cells. Assessment of expression of luciferase vectors of a pathway reporter array in 2-fold serial dilution cultures, as ascertained by luciferase assay $48 \mathrm{~h}$ after cell seeding, showing fold change in cell expression relative to control; the corresponding control cultures were seeded with $3,700 \mathrm{cell} / \mathrm{s} / \mathrm{cm}^{2}$. As cell confluency increased, both the transcription of Elk-1/SRF- and RBPJ-responsive vectors exceeded the activation threshold (dashed line), indicating that both transcription factors activated transcription as a result of intercellular adhesion. The ratios of total protein between consecutive cultures in the series (seeding ratio, 2) were ascertained by protein determination $48 \mathrm{~h}$ after cell seeding, obtaining ratios equal to the seeding ratio. The points and bars represent average and SD values, respectively, of 3 different experiments.

\section{Results}

Intercellular integrin binding activates both ERK and Notch target transcription in U87MG glioblastoma cells. The assessment of transcription of vectors of a pathway reporter array in 2 -fold serial dilution cultures revealed both the activation of Elk-1/SRF- and RBPJ-driven transcription in the cell cultures at $32 \%$ confluency and further enhanced transcription in the cell cultures at $64 \%$ confluency (Fig. 1), indicating that intercellular adhesion elicited signal transduction through either extracellular signal-regulated kinase (ERK), Notch or both pathways. Given that cell adhesion-induced ERK activation is determined by integrin binding to protein ligands (11), we subsequently assessed cell density-dependent transcriptional activation in cultures containing integrin- $\beta 1$ antibody. Antibody targeting of integrin- $\beta 1$ hindered the induction of both ERK and Notch target transcription (Table I), suggesting that integrin-mediated signal transduction activated ERK and that, concomitantly, there was either a crosstalk between the ERK and Notch signaling pathways or an interaction between the integrin and Notch receptors (12). Since Nr-CAM is a binding partner of integrins in cell-cell adhesion (13), we also determined cell density-dependent transcriptional activation in cultures containing a specific antibody for that adhesion receptor. This corroborated that signaling decreased (Table I) upon the antibody targeting of a ligand of integrins at the 
Table I. Decreased signaling in U87MG glioblastoma cell cultures upon antibody targeting of integrin- $\beta 1$ or $\mathrm{Nr}-\mathrm{CAM}$.

Fold increase in transcription

factor-regulated expression relative to the control ${ }^{\mathrm{a}}$

\begin{tabular}{lcc}
\cline { 2 - 3 } Antibody & Elk-1/SRF & RBPJ \\
\hline Non-specific & $1.8 \pm 0.4$ & $2.0 \pm 0.2^{\mathrm{S}}$ \\
Integrin- $\beta 1$ & $1.0 \pm 0.1^{\mathrm{b}}$ & $0.8 \pm 0.2^{\mathrm{c}}$ \\
Nr-CAM & $0.9 \pm 0.2^{\mathrm{b}}$ & $1.1 \pm 0.2^{\mathrm{c}}$ \\
Hyaluronan receptor & $1.8 \pm 0.2$ & $1.6 \pm 0.3$
\end{tabular}

${ }^{\text {a}}$ Fold change in cell expression of reporter in cultures at $32 \%$ confluency (seeding density, 29,600 cells $/ \mathrm{cm}^{2}$ ), containing either cells with Elk-1/ SRF-responsive vector or cells with RBPJ-responsive vector, relative to the control, as ascertained by luciferase assay $48 \mathrm{~h}$ after cell seeding. The corresponding control cultures were seeded with 3,700 cells $/ \mathrm{cm}^{2}$; all cultures contained antibody at 1:500 dilution. Values are the means \pm SD of 3 different experiments; ${ }^{b} p<0.05$ or ${ }^{c} p<0.01$, as shown by the Student's t-test. Nr-CAM, neuronal cell adhesion molecule.

Table II. Induction of both ERK and Notch target transcription in U87MG cells by integrin-mediated adhesion to retronectin coating.

Fold change in transcription factor-regulated

Retronectin concentration $\left(\mu \mathrm{g} / \mathrm{cm}^{2}\right)$ expression relative to the control ${ }^{\mathrm{a}}$

\begin{tabular}{lll}
\hline 8.0 & $0.9 \pm 0.1$ & \\
4.5 & $1.0 \pm 0.2$ & $1.1 \pm 0.2$ \\
3.0 & $1.0 \pm 0.1$ & $0.9 \pm 0.3$ \\
1.5 & $0.9 \pm 0.1$ & $1.1 \pm 0.3$ \\
0.5 & $1.4 \pm 0.1^{\mathrm{b}}$ & $1.0 \pm 0.1$ \\
\hline
\end{tabular}

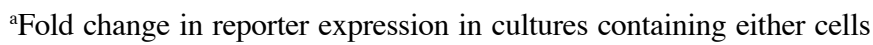
with Elk-1/SRF-responsive vector or cells with RBPJ-responsive vector relative to the control, as ascertained by luciferase assay $24 \mathrm{~h}$ after cell seeding; the corresponding control cultures were seeded on surface coated with $15.0 \mu \mathrm{g}$ retronectin $/ \mathrm{cm}^{2}$. Seeding density, $15,000 \mathrm{cell} / \mathrm{s} / \mathrm{cm}^{2}$; values are the means \pm SD of 3 different experiments; ${ }^{b}$ significantly different $(\mathrm{p}<0.01)$ as shown by the Student's t-test from values obtained on coating with $8.0 \mu \mathrm{g}$ retronectin $/ \mathrm{cm}^{2}$.

cell surface. Conversely, there was signaling activation in the cultures containing non-specific antibody or blocking antibody for hyaluronan receptor (Table I), a mediator of cell-cell adhesion that has been related to cell fusion $(7,14)$, which indicated that cell confluence and signal transduction were not impaired by the side-effects of the antibody. In summary, these results revealed that intercellular integrin binding elicited adhesion signaling in U87MG cells that activated both ERK and Notch target transcription.
Table III. U87MG cell cultures on coating that promoted integrin adhesion signaling show enhanced frequency of polynucleation.

\begin{tabular}{lcccc}
\hline \multicolumn{2}{c}{$15.0 \mu \mathrm{g}$ Retronectin/cm } & & & $0.5 \mu \mathrm{g}$ Retronectin $/ \mathrm{cm}^{2}$ \\
\cline { 1 - 1 } \cline { 5 - 5 } Binucleated/total cells & $\%$ & & Binucleated/total cells & $\%$ \\
\hline $22 / 388$ & 6 & & $38 / 361$ & 11 \\
$11 / 236$ & 5 & & $31 / 315$ & 10 \\
$31 / 458$ & 7 & & $32 / 283$ & 11 \\
$35 / 390$ & 9 & & $31 / 292$ & 11 \\
$13 / 278$ & 5 & & $56 / 334$ & 17 \\
$22 / 334$ & 7 & & $22 / 271$ & 8 \\
Means \pm SD & $7 \pm 2$ & & $11 \pm 3^{\mathrm{a}}$ \\
\hline
\end{tabular}

${ }^{\text {a }}<00.01$, as shown by the Student's t-test.

Enhanced frequency of protease-driven polyploidization upon activation of both ERK and Notch target transcription. We assessed the level of both ERK and Notch target transcription following integrin-based cell adhesion to surfaces coated with retronectin, a recombinant adhesion protein with integrin binding sites of fibronectin, which is a pericellular matrix component robustly expressed by U87MG cells (15). Cell attachment to coating at a concentration of $0.5 \mu \mathrm{g} / \mathrm{cm}^{2}$ involved transcriptional activation (Table II), providing the induction of both ERK and Notch target transcription. We then determined the rate of proliferation and the percentage of cells with a 4-fold increase in the amount of cellular DNA, corresponding to the G2/M peak of the cells with duplicated tumor genome (9), of cultures on coating that promoted integrin adhesion signaling. The fold increase in the number of cells in the 24-48 h interval from cell seeding, $2.8 \pm 0.9$, was almost equal to that of the control cultures $(15 \mu \mathrm{g}$ retronectin $/ \mathrm{cm}^{2}$ ), $2.9 \pm 0.7$ (means \pm SD of 3 different experiments). The relative number of cells with duplicated tumor genome, by contrast, increased significantly (Fig. 2). In agreement with this result, the cultures contained a markedly enhanced proportion of binucleated cells (Table III). This linked the transcriptional activation induced by integrin adhesion to an increased rate of cell polyploidization. Hence, we quantified the cells with a duplicated tumor genome in regular cultures containing antibody that prevented signaling activation. The cultures containing antibody for hyaluronan receptor, which prevented the polyploidization of breast carcinoma and melanoma cells, showed an unaltered frequency of polyploidization (Table IV). By contrast, the cultures containing integrin- $\beta 1$ antibody showed a reduced frequency (Table $\mathrm{V}$ ). These findings indicated that the induction of both ERK and Notch target transcription resulted in tumor-genome duplication.

Of note, the U87MG cells on coating that promoted integrin adhesion signaling extended thin protruding adhesive structures resembling matrix-degrading invadopodia (Fig. 3), which suggested that the induction of genome duplication was mediated by intracellular invasion. Hence, we quantified the cells with a duplicated tumor genome in the regular cell cultures containing specific inhibitors of cancer cell invasion-associated MMPs. Cultures containing MMP inhibitors had markedly low percentages of polyploid cells (Table VI). Since these findings 
A $15.0 \mu \mathrm{g}$ retronectin $/ \mathrm{cm}^{2}$
(control)

S-phase peak: 442 cells

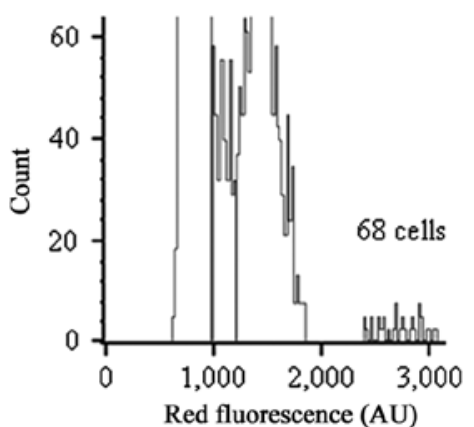

$0.5 \mu \mathrm{g}$ retronectin $/ \mathrm{cm}^{2}$

S-phase peak: 412 cells

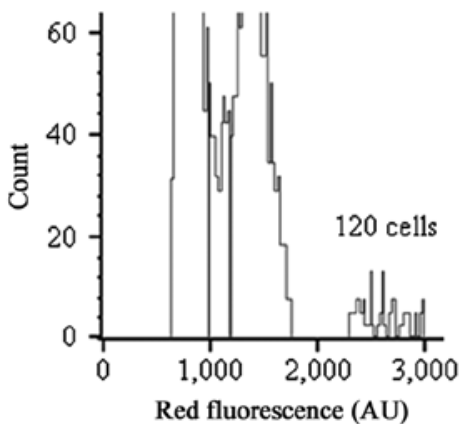

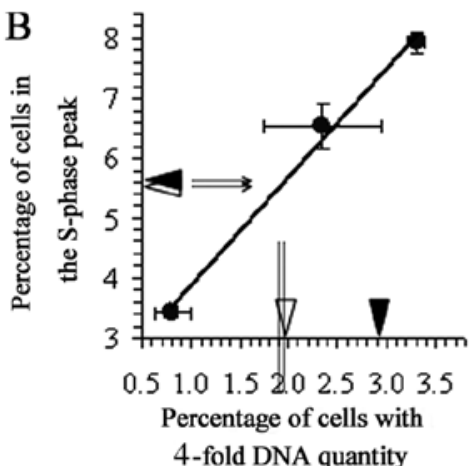

Deviations from correlation (mean $\pm \mathrm{SD}, \mathrm{n}=6$ )

$0.1 \pm 0.7$ (control)

$1.0 \pm 0.3 *$

Figure 2. U87MG cell cultures on coating that promoted integrin adhesion signaling show increased frequency of tumor-genome duplication. (A) Cell DNA content distribution analyses of 3-day cultures on retronectin-coated surface revealing an increase in the proportion of cells with a 4-fold increase in the amount of DNA. (B) Correlation in regular cultures between percentage of cells in the S-phase peak and percentage of cells with a 4-fold increase in the amount of DNA $\left(r^{2}>0.95\right)$; each point represents the average obtained from cultures with nearly equal percentage of cells in S-phase; bars represent SD values. Average values obtained from 3-day cultures on surface coated with 15 (control) or $0.5 \mu \mathrm{g}$ retronectin/ $\mathrm{cm}^{2}$, indicated by white and black arrowheads, respectively, revealed a statistically significant deviation from the correlation in the percentage of cells with a 4-fold increase in the amount of DNA. Arrowheads indicate the average of 2 different experiments (seeding density, 15,000 cells $/ \mathrm{cm}^{2}$ ); ${ }^{*} \mathrm{p}<0.05$, as shown by the Student's t-test; AU, arbitrary units.
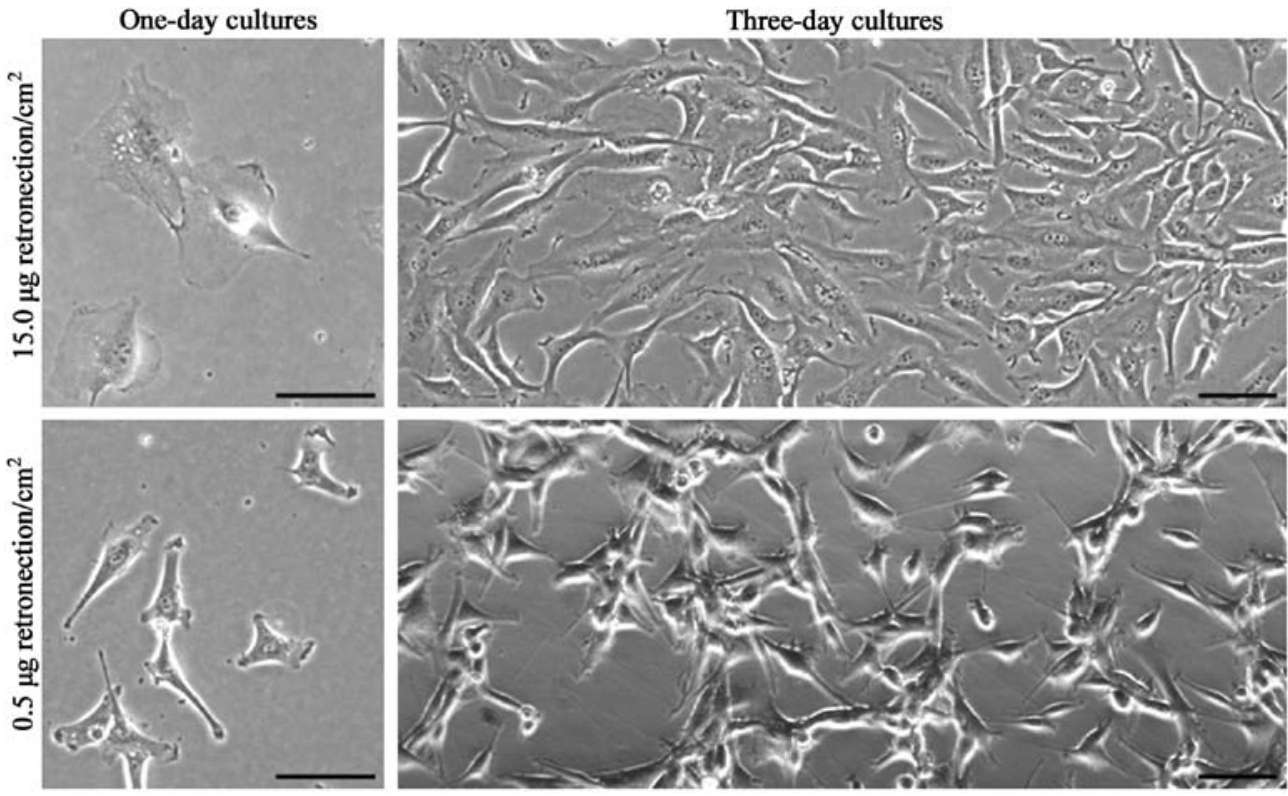

Figure 3. Micrographs showing differing U87MG cell morphologies after attachment to coating that did not promote integrin adhesion signaling (15 $\mu \mathrm{g}$ retronectin $\left./ \mathrm{cm}^{2}\right)$, flat morphology and wide cell extensions, or coating that promoted integrin adhesion signaling $\left(0.5 \mu \mathrm{g}\right.$ retronectin $\left./ \mathrm{cm}^{2}\right)$, rounded morphology and thin podosome-like adhesive structures. Seeding density, 15,000 cells $/ \mathrm{cm}^{2} ;$ bars, $0.1 \mathrm{~mm}$.

indicated that adhesion-stimulated genome duplication was mediated by protease-driven tumor invasion, we wished to investigate whether intracellular invasion by U87MG cells resulted in the merging of cell contents. In co-cultures in non-coated dishes of U87MG cells expressing red fluorescent protein and non-tumor neural cells expressing green fluorescent protein, intracellular fluorescence was observed that revealed that the cytoplasmic content of tumor cells was released through fusion pores. Representative images are presented in Fig. 4. These observations revealed that intracellular invasion by U87MG cells resulted in the merging of cell contents, further suggesting that tumor invasion mediated the increases in DNA content.

\section{Discussion}

In the present study, we found that signal transduction promoted the polyploidization of U87MG glioblastoma cells. Specifically, the activation of both ERK and Notch target transcription induced by integrin adhesion resulted in a significantly enhanced number of hypotetraploid cells. This indicated that adhesion-stimulated polyploidization was associated with the 


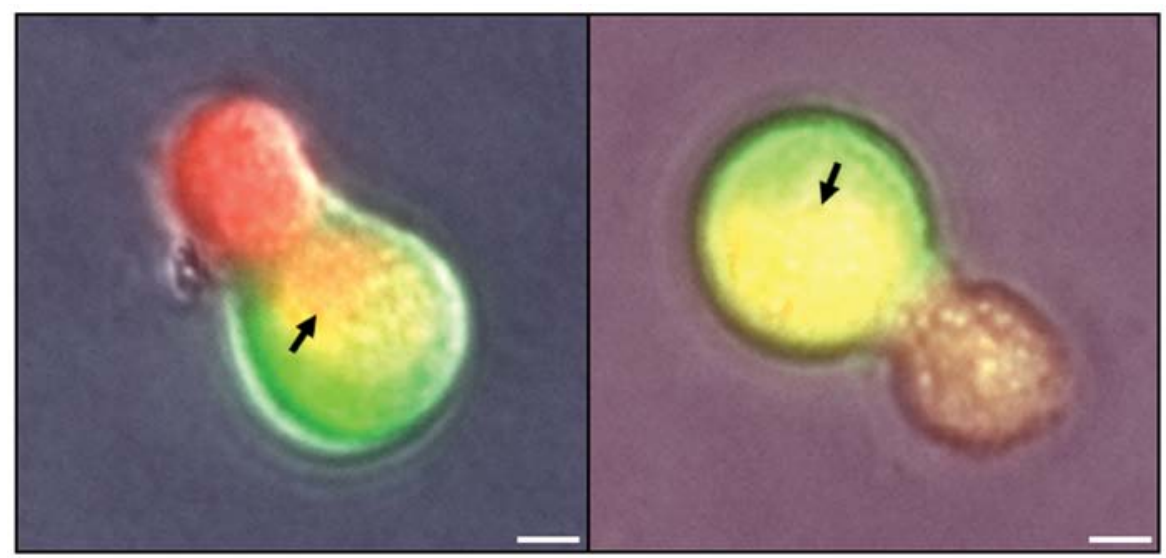

Figure 4. Intracellular invasion by U87MG glioblastoma cells. Fluorescent light micrographs of cell doublets composed of a U87MG cell and a non-tumor neural cell expressing red and green fluorescent protein, respectively, showing merging of cell contents (arrows). Bars, $5 \mu \mathrm{m}$.

Table IV. Despite expressing hyaluronan receptor, as determined by analysis using phycoerythrin-conjugated antibody, U87MG glioblastoma cell cultures containing specific antibody show unchanged frequency of polyploidization ${ }^{\mathrm{a}}$.

\begin{tabular}{|c|c|c|c|c|c|c|}
\hline \multirow[b]{2}{*}{ Antibody } & \multicolumn{3}{|c|}{ Fluorescence intensity (a.u.) } & \multicolumn{3}{|c|}{$\begin{array}{l}\text { Cells with 4-fold increase in DNA quantity } \\
\text { (percentage of control) }\end{array}$} \\
\hline & U87MG & MDA & FEMX & U87MG & MDA & FEMX \\
\hline Non-specific & $2 \pm 1$ & $2 \pm 1$ & $1 \pm 0$ & $95 \pm 23$ & $100 \pm 19$ & $99 \pm 16$ \\
\hline Hyaluronan receptor & $831 \pm 268$ & $827 \pm 261$ & $393 \pm 105$ & $106 \pm 9$ & $58 \pm 7^{c}$ & $51 \pm 22^{\mathrm{c}}$ \\
\hline
\end{tabular}

Table V. Reduced proportion of cells with a 4-fold increase in DNA quantity in U87MG cell cultures containing antibody for integrin- $\beta 1$.

\begin{tabular}{lc} 
Antibody & $\begin{array}{c}\text { Cells with 4-fold increase in } \\
\text { DNA quantity (percentage of control) }\end{array}$ \\
\hline Non-specific & $109 \pm 19$ \\
Integrin- $\beta 1$ & $77 \pm 13^{\mathrm{b}}$ \\
\hline
\end{tabular}

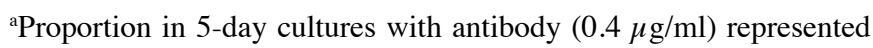
as a percentage of the average of cultures without antibody, as determined by cell DNA content distribution analysis. Values are the means \pm SD of 3 different experiments; ${ }^{b} \mathrm{p}<0.05$, as shown by the Student's t-test.

activation of signaling networks that regulate the expression of proteolytic enzymes that elicit invasion, since Notch signaling regulates the expression of adamalysin proteins (16), which are reportedly profusogenic metalloproteases $(6,8)$ and ERK signaling involves the transcriptional control of MMP expression (17-20). Accordingly, inhibitors for the specific
Table VI. Reduced proportion of cells with a 4-fold increase in DNA quantity in U87MG cell cultures containing MMP inhibitor.

\begin{tabular}{lc} 
Compound & DNA quantity (percenta \\
\hline Chemical solvent & $100 \pm 20$ \\
MMP inhibitor II & $59 \pm 19^{\mathrm{b}}$ \\
Marimastat & $62 \pm 6^{\mathrm{b}}$
\end{tabular}

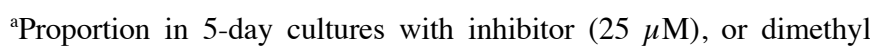
sulfoxide at the corresponding concentration $(<0.1 \%)$, represented as a percentage of the average of compound-free cultures, as determined by cell DNA content distribution analysis. Values are the means $\pm \mathrm{SD}$ of 3 different experiments; ${ }^{b} \mathrm{p}<0.01$, as shown by the Student's t-test. MMP, matrix metalloproteinase.

blockage of several types of cancer cell invasion-associated MMPs markedly reduced the frequency of tumor-genome duplication. Thus, consistent with the observation that intracellular invasion by U87MG cells resulted in the merging of cell contents, both the finding that transcriptional activation and podosome-like adhesive structures appeared together, 
and the inhibitory effect of MMP blockage revealed that the induction of tumor-genome duplication was mediated by protease-driven invasion. Our results therefore indicated that the activation of cell invasion-regulating signaling networks determined the appearance of polyploid cells.

Both the antibody targeting of $\mathrm{Nr}-\mathrm{CAM}$, a ligand of integrins in cell-cell adhesion that is overexpressed in human brain tumors (21) and integrin- $\beta 1$, a molecule that also mediates integrin receptor adhesion to matrix proteins (11), hindered signaling activation in U87MG cell cultures. This suggested that both intercellular integrin binding to proteins of the plasma membrane and pericellular matrix elicited the transduction of signals that induced polyploidization. We previously found that the antibody targeting of hyaluronan receptor significantly reduced the proportion of polyploid cells in both breast carcinoma and melanoma cell cultures (9), thus supporting a role of that adhesion receptor in cancer invasion-mediated cell fusion. In this study, by contrast, the antibody did not hinder the polyploidization of glioblastoma cells. As the expression of hyaluronan receptor on the cell surface was not exceedingly high, this result seemed unrelated to the level of receptor saturation reached by the antibody. Instead, the lack of inhibitory effect suggested that intercellular interactions of hyaluronan receptor did not result in polyploidization.

In conclusion, the findings of the present study demonstrate the association of the activation of invasion signaling upon cell adhesion with the appearance of tumor-genome duplication, supporting cell invasiveness as a cause of ploidy turnover in tumor cell populations.

\section{References}

1. Sluder G and Nordberg JJ: The good, the bad and the ugly: the practical consequences of centrosome amplification. Curr Opin Cell Biol 16: 49-54, 2004.

2. Davoli T and de Lange T: The causes and consequences of polyploidy in normal development and cancer. Annu Rev Cell Dev Biol 27: 585-610, 2011.

3. Vitale I, Galluzzi L, Senovilla L, Criollo A, Jemaà M, Castedo M and Kroemer G: Illicit survival of cancer cells during polyploidization and depolyploidization. Cell Death Differ 18: 1403-1413, 2011.

4. Chakraborty AK, Sodi S, Rachkovsky M, Kolesnikova N, Platt JT, Bolognia JL and Pawelek JM: A spontaneous murine melanoma lung metastasis comprised of host $\mathrm{x}$ tumor hybrids. Cancer Res 60: 2512-2519, 2000

5. Lagarde AE, Donaghue TP, Dennis JW and Kerbel RS Genotypic and phenotypic evolution of a murine tumor during its progression in vivo toward metastasis. J Natl Cancer Inst 71: $183-191,1983$
6. Ogle BM, Cascalho M and Platt JL: Biological implications of cell fusion. Nat Rev Mol Cell Biol 6: 567-575, 2005.

7. Chen EH, Grote E, Mohler W and Vignery A: Cell-cell fusion. FEBS Lett 581: 2181-2193, 2007.

8. Zhou X and Platt JL: Molecular and cellular mechanisms of mammalian cell fusion. Adv Exp Med Biol 713: 33-64, 2011.

9. Mercapide J, Anzanello F, Rappa G and Lorico A: Relationship between tumor cell invasiveness and polyploidization. PLoS One 7: e53364, 2012

10. Lorico A, Mercapide J, Solodushko V, Alexeyev M, Fodstad O and Rappa G: Primary neural stem/progenitor cells expressing endostatin or cytochrome P450 for gene therapy of glioblastoma. Cancer Gene Ther 15: 605-615, 2008.

11. Yee KL, Weaver VM and Hammer DA: Integrin-mediated signalling through the MAP-kinase pathway. IET Syst Biol 2: $8-15,2008$.

12. Campos LS, Decker L, Taylor V and Skarnes W: Notch, epidermal growth factor receptor, and beta1-integrin pathways are coordinated in neural stem cells. J Biol Chem 281: 5300-5309, 2006.

13. Conacci-Sorrell M, Kaplan A, Raveh S, Gavert N, Sakurai T and Ben-Ze'ev A: The shed ectodomain of Nr-CAM stimulates cell proliferation and motility, and confers cell transformation. Cancer Res 65: 11605-11612, 2005.

14. Sterling H, Saginario C and Vignery A: CD44 occupancy prevents macrophage multinucleation. J Cell Biol 143: 837-847, 1998.

15. Enam SA, Rosenblum ML and Edvardsen K: Role of extracellular matrix in tumor invasion: migration of glioma cells along fibronectin-positive mesenchymal cell processes. Neurosurgery 42 : 599-607, 1998

16. Díaz B, Yuen A, Iizuka S, Higashiyama $S$ and Courtneidge SA: Notch increases the shedding of HB-EGF by ADAM12 to potentiate invadopodia formation in hypoxia. J Cell Biol 201: 279-292, 2013

17. Anand M, van Meter TE and Fillmore HL: Epidermal growth factor induces matrix metalloproteinase-1 (MMP-1) expression and invasion in glioma cell lines via the MAPK pathway. J Neurooncol 104: 679-687, 2011.

18. Kunapuli P, Kasyapa CS, Hawthorn L and Cowell JK: LGI1, a putative tumor metastasis suppressor gene, controls in vitro invasiveness and expression of matrix metalloproteinases in glioma cells through the ERK1/2 pathway. J Biol Chem 279: 23151-23157, 2004

19. Lakka SS, Jasti SL, Gondi C, Boyd D, Chandrasekar N, Dinh DH, Olivero WC, Gujrati M and Rao JS: Downregulation of MMP-9 in ERK-mutated stable transfectants inhibits glioma invasion in vitro. Oncogene 21: 5601-5608, 2002.

20. Lin YM, Jan HJ, Lee CC, Tao HY, Shih YL, Wei HW and Lee HM: Dexamethasone reduced invasiveness of human malignant glioblastoma cells through a MAPK phosphatase-1 (MKP-1) dependent mechanism. Eur J Pharmacol 593: 1-9, 2008.

21. Sehgal A, Boynton AL, Young RF, Vermeulen SS, Yonemura KS Kohler EP, Aldape HC, Simrell CR and Murphy GP: Cell adhesion molecule $\mathrm{Nr}$-CAM is over-expressed in human brain tumors. Int J Cancer 76: 451-458, 1998. 